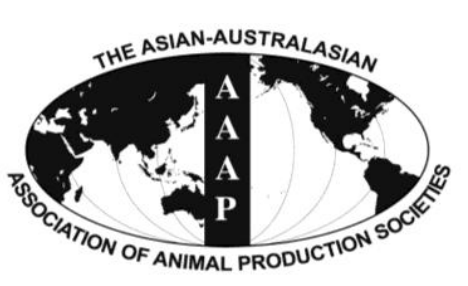

\title{
Molecular Characterization and Expression Analysis of Insulin-like Growth Factor-1 and Insulin-like Growth Factor Binding Protein-1 Genes in Qinghai-Tibet Plateau Bos grunniens and Lowland Bos taurus
}

\author{
Ya-bing Chen ${ }^{1, a}$, Mei Fu ${ }^{1, a}$, Dao-liang $\operatorname{Lan}^{2}$, and Jian $\mathrm{Li}^{1,2, *}$ \\ ${ }^{1}$ College of Life Science and Technology, Southwest University for Nationalities, \\ Chengdu, Sichuan 610041, China
}

\begin{abstract}
Insulin-like growth factor-1 (IGF-1) and insulin-like growth factor binding protein-1 (IGFBP-1) play a pivotal role in regulating cellular hypoxic response. In this study, we cloned and characterized the genes encoding IGF-1 and IGFBP-1 to improve the current knowledge on their roles in highland Bos grunniens (Yak). We also compared their expression levels in the liver and kidney tissues between yaks and lowland cattle. We obtained full-length 465 bp IGF-1 and 792 bp IGFBP-1, encoding 154 amino acids (AA) IGF-1, and 263 AA IGFBP-1 protein, respectively using reverse transcriptase-polyerase chain reaction (RT-PCR) technology. Analysis of their corresponding amino acid sequences showed a high identity between B. grunniens and lowland mammals. Moreover, the two genes were proved to be widely distributed in the examined tissues through expression pattern analysis. Real-time PCR results revealed that IGF-1 expression was higher in the liver and kidney tissues in B. grunniens than in Bos taurus ( $\mathrm{p}<0.05$ ). The IGFBP-1 gene was expressed at a higher level in the liver $(\mathrm{p}<0.05)$ of B. taurus than B. grunniens, but it has a similar expression level in the kidneys of the two species. These results indicated that upregulated IGF-1 and downregulated IGFBP-1 are associated with hypoxia adaptive response in B. grunniens. (Key Words: Bos grunniens, Insulin-like Growth Factor-1, Insulin-like Growth Factor Binding Protein-1, Cloning, Gene expression)
\end{abstract}

\section{INTRODUCTION}

The insulin-like growth factor (IGF) superfamily, is a functionally diverse protein family that mediates in the metabolism, growth, and differentiation of mammals. The family consists of two ligands (IGF1 and IGF2), two receptors (IGF1R and IGF2R), and six binding proteins (insulin-like growth factor binding protein [IGFBP1 to IGFBP6])(Annunziata et al., 2011). The IGF1 is a small peptide hormone and responsible for mammalian metabolism, growth, and development (Annunziata et al.,

\footnotetext{
* Corresponding Author: Jian Li. Tel: +86-028-8552-2227,

E-mail: lijian@swun.cn

${ }^{2}$ Institute of Qinghai-Tibetan Plateau, Southwest University for Nationalities, Chengdu, Sichuan 610041, China.

a These authors contributed equally to this work.

Submitted May 22, 2014; Revised Aug. 7, 2014; Accepted Aug. 18, 2014
}

2011). Furthermore, IGF-1 can induce the expression of hypoxia inducible factor (HIF1) under hypoxic or ischemic condition, triggering the expression of various target genes that regulate the cellular hypoxic response (Lee et al., 1997; Sugawara et al., 2000; Fukuda et al., 2002; Rajpathak et al., 2009). The IGFBP-1, a key modulator protein of the IGF system, is mainly synthesized in hepatocytes and renal cells and released from the liver into the circulation to bind to IGF-1 with higher affinity than its receptor IGFR1 to govern IGF-1 bioavailability and action. Moreover, a growing body evidence has shown that IGFBP-1 plays an important role in hypoxic response through adjusting the availability of IGF1 in circulation, influencing its downstream reaction (Jones and Clemmons, 1995; Annunziata et al., 2011; Sun et al., 2011; Yu et al., 2012).

In recent years, the adaptation of plateau mammals to high-altitude hypoxia has attracted increasing attention. 
Numerous species on the Tibet plateau have been examined to explore the hypoxia-related molecular mechanisms (Wei et al., 2006; Cao et al., 2008; Cao et al., 2009; Zhang et al., 2013), but little information about yaks is available. Yak (Bos grunniens) is the most important animal species in the Qinghai-Tibet plateau not only because of its economic value in meat, milk, and usage in agriculture, but also as an ideal model animal for studying the molecular mechanisms of adapting to the cold and low-oxygen environment (Hu et al., 2012). By contrast, closely related and cross-fertile cattle (Bos taurus) suffer from severe pulmonary hypertension when reared in the same habitat (Weir et al., 1974; Will et al., 1975; Hu et al., 2012). Yaks have numerous anatomical and physiological traits that equip them for life at high altitude. Therefore, this highland animal is an ideal model animal for studying the mechanisms of adapting to cold and low-oxygen environments. In this study, we compared the variation in the nucleotide sequences and mRNA expression levels of IGF-1 and IGFBP-1 genes in the yak and lowland cattle to examine whether base mutations or expression levels of genes might be associated with the difference in adaptation to hypoxia.

\section{MATERIALS AND METHODS}

\section{Sample collection and preservation}

The yaks were purchased from the Ganzi district (3500 $\mathrm{m}$ in altitude), Sichuan, China, and cattle were obtained from the Guanghan county (450 $\mathrm{m}$ in altitude). Several tissues from the heart, liver, spleen, lung, kidney, large intestine, small intestine, stomach, muscle, mammary gland, ovary, testis, and brain were collected through slaughter and were immediately frozen in liquid nitrogen until future use. All experimental procedures were performed according to Guidelines on the Care and Use of Laboratory Animals of the Institutional Animal Care and Use Committee of Southwest University for Nationalities, Sichuan, China.

\section{RNA isolation and reverse transcription-polymerase chain reaction}

RNAs were isolated using Trizol (Invitrogen, Shanghai, China) according to the manufacturer's instructions. Reverse transcription was performed using an RT reagent kit (Takara, Dalian, China). The generated cDNA was amplified using gene specific primers (IGF1:5'AATGGGAAAAATCAGCAGTCTT -3'/5' - TTCTGTAG TTCTTGTTTCCTGCA -3'; IGFBP1: 5'- TTCCTGTC TGCCACCCA -3'/5'- TTGCATCTGTTTTCAGTTCTG 3') designed based on the conserved regions of corresponding cDNAs among sheep, goat, and cattle available in the GenBank database. The purified PCR products were cloned into PMD-19T vectors (Takara,
China) and sequenced.

\section{Bioinformatics analysis}

The sequences of cloned genes were subjected to basic local alignment search tool analysis for verification. The identity of $I G F-1$ and $I G F B P-1$ gene between yak and other mammals were analyzed using online Multalin software.

\section{Tissue expression patterns of $I G F-1$ and $I G F B P-1$ genes}

The expression patterns of $I G F-1$ and $I G F B P-1$ genes in the different tissues were investigated using semiquantitative reverse transcription-polymerase chain reaction (RT-PCR) on cDNAs obtained from the different tissues, and the $\beta$-actin gene was chosen as the positive control. PCRs were performed using special primers (IGF: 5'GTGCGGAGACAGGGGCTTT-3'/5' - ACTTGGCGGGC TTGAGAGG-3'; IGFBP1: 5' - CACTTGATGGCCGAGT CCA-3'/5'- CCTCCAGCGACGTCTCACA-3'; $\beta$-actin: 5'AGGTGACAGCAGTCGGTTGGA-3'/5'--CCTTAGAGA GAAGCGGGGTGG -3').

\section{Quantitative real-time polymerase chain reaction}

Considering that the IGF-1 and IGFBP-1 in the blood are predominately produced in the liver and kidney, the expression levels of IGF1 and IGFBP1 between the yak and cattle were compared. Moreover, $\beta$-actin gene was chosen to normalize the expression levels of the target genes. Realtime PCR was performed using a CFX-manger connect real-time detection system (Bio-Rad, Hercules, CA, USA) with SYBR Premix Ex-Taq TM II (Takara, China). All samples were ran in triplicate, and the gene expression level was calculated according to the $2^{-\Delta \mathrm{Ct}}$ method (Schmittgen and Livak, 2008). Statistical differences were analyzed using Student's $t$-test, and the variance was considered significant when $\mathrm{p}<0.05$.

\section{RESULTS AND DISCUSSIONS}

\section{Molecular characterization of cDNAs for IGF-1 and IGFBP-1}

The cloned sequences of IGF-1 and IGFBP-1 were 468 and $792 \mathrm{bp}$, respectively, and sequences were submitted to the GenBank under Accession Nos. KJ489376 and KJ489377, respectively. The $I G F-1$ gene was predicted to encode a peptide of 154 amino acid residues, including a signal peptide (49 residues), a mature peptide (70 residues), and a 35-residue C-terminal extension (E). Four characteristic domains of IGF-1 (B, C, A, and D) were also predicted in the mature peptide, based on its conservation among mammals (Figure 1A). Compared with the other mammals, no special variation was detected in the IGF-1 mature peptide. The IGFBP-1 gene encodes a peptide of 263 amino-acid residues, composed of $\mathrm{N}$ domain, central 


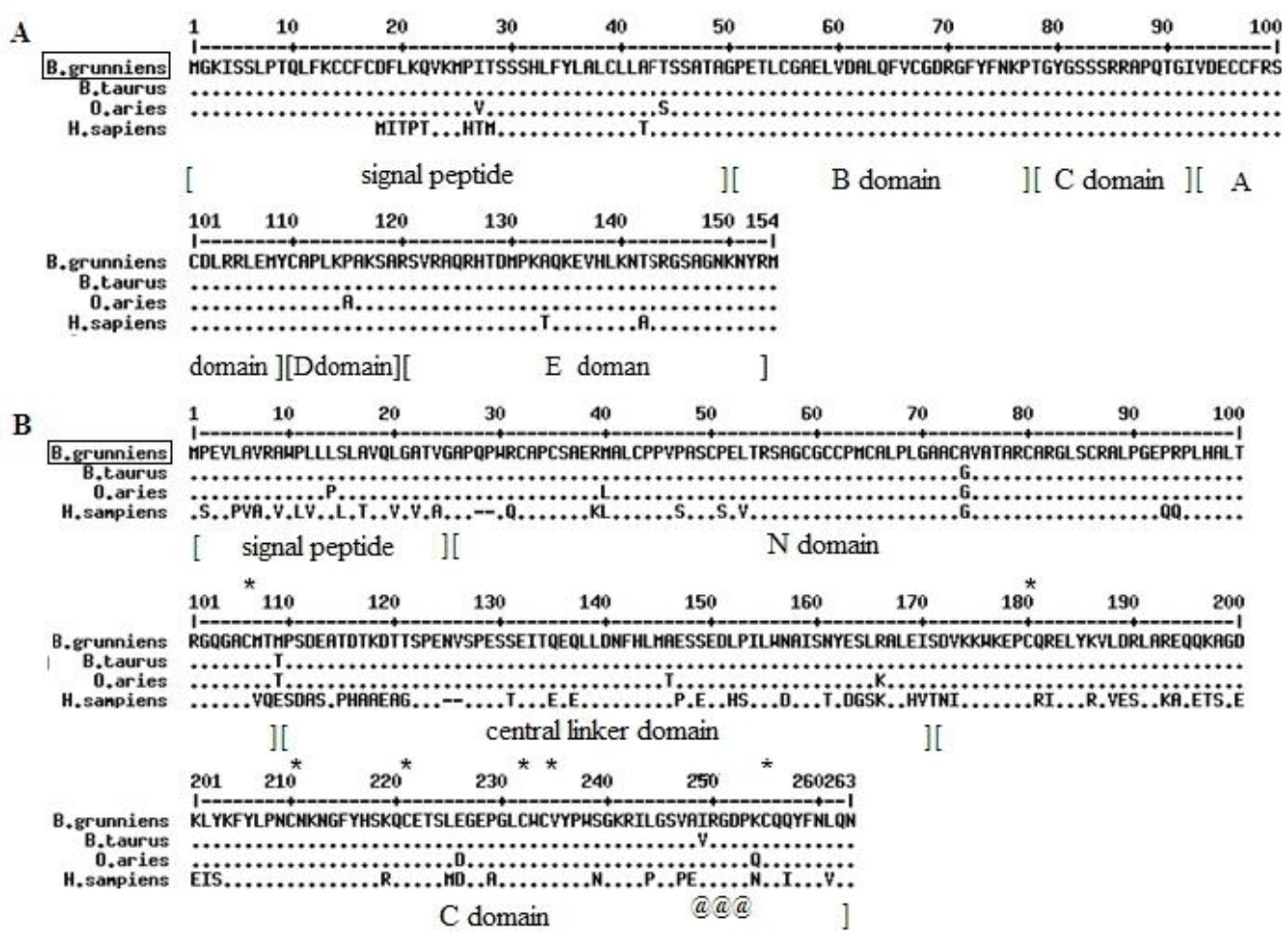

Figure 1. Alignment of deduced amino acid sequences of $I G F-1$ and $I G F B P-1$ genes. The peptide of (A) IGF-1 and (B) IGFBP-1 were compared with the corresponding sequences from B. taurus, $O$. aries, and $H$. sapiens. Conserved sequences are indicated by $(\bullet)$, and the variant residues were listed. The predicted domains of the IGF-1 (signal peptide, B domain, C domain, A domain, D domain, E domain) and IGFBP-1 (signal peptide, $\mathrm{N}$ domain, central linker domain, $\mathrm{C}$ domain) were also marked. The highly conserved cysteines in the IGFBP-1 were indicated by “*”, and the characteristic RGD motif in the C domain was indicated by “@”. IGF-1, insulin-like growth factor-1; IGFBP-1, insulin-like growth factor binding protein-1; RGD, arginine-glycine-aspartic acid.

linker domain, and $\mathrm{C}$ domain (Figure 1B). The $\mathrm{N}$ and $\mathrm{C}$ domains, which are rich in cysteine and responsible for IGF binding, were highly conserved in $B$. grunniens. Additionally, the arginine - glycine - aspartic acid motif in the $\mathrm{C}$ domain, which was verified to be involved in IGFindependent effect, was also examined in B. grunniens. Thus, IGF- 1 and IGFBP- 1 genes from B. grunniens shared high homology with those of B. taurus, Ovis aries, and homo sapiens in the nucleotide and protein level, which suggests that the base mutations of the two target genes do not induce the different resistances to hypoxia.

\section{Tissue distribution analysis of IGF-1 and IGFBP-1}

Semi-quantitative RT-PCR was performed to examine the distribution patterns of IGF-1 and IGFBP-1 in bovines. The IGF-1 and IGFBP-1 transcripts were detected in all tissues examined (Figure 2). Higher levels of IGF-1 transcript were noted in the heart, liver, spleen, kidney, large intestine, stomach, muscle, and brain. Higher levels of IGFBP-1 transcript were detected in the liver, kidney, muscle, brain. These results are in accordance with the previous finding that the IGF-1 and IGFBP-1 are synthesized mainly in the liver and released into the circulation to regulate growth and development (Ohlsson et al., 2009). In addition, the widespread distribution of IGF-1 and IGFBP-1 supported their varied physiological roles in animals.

\section{Variation in the mRNA expression levels of IGF-1 and IGFBP-1 in Tibet plateau yaks and lowland cattle}

The IGF-1 is a hormone that regulates the growth of vertebrates and can protect cells against certain type of stress. The IGF-1 can improve the survival of cells under hypoxic or ischemic conditions (Mehrhof et al., 2001; Eliasz et al., 2010). Studies have shown that IGF-1 in circulation can exert its biological effects through binding to and activating the transmembrane receptors (Murphy and

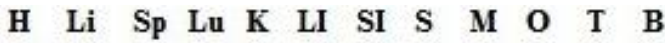

\section{IGF1 \\ IGFBPI}

B-actin

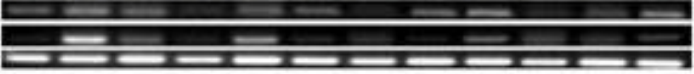

Figure 2. Tissue distribution of the gene expression of IGF-1 and IGFBP-1. RT-PCR was performed on the cDNA from the yak, and the $\beta$-actin gene was chosen as the positive control. $\mathrm{H}$, heart; $\mathrm{Li}$, liver; S, spleen; Lu, lung; K, kidney; LI, large intestine; SI, small intestine; S, stomach; M, muscle; O, ovary; T, testis; B, brain; IGF-1, insulin-like growth factor-1; IGFBP-1, insulin-like growth factor binding protein-1; RT-PCR, reverse transcriptase-polyerase chain reaction. 

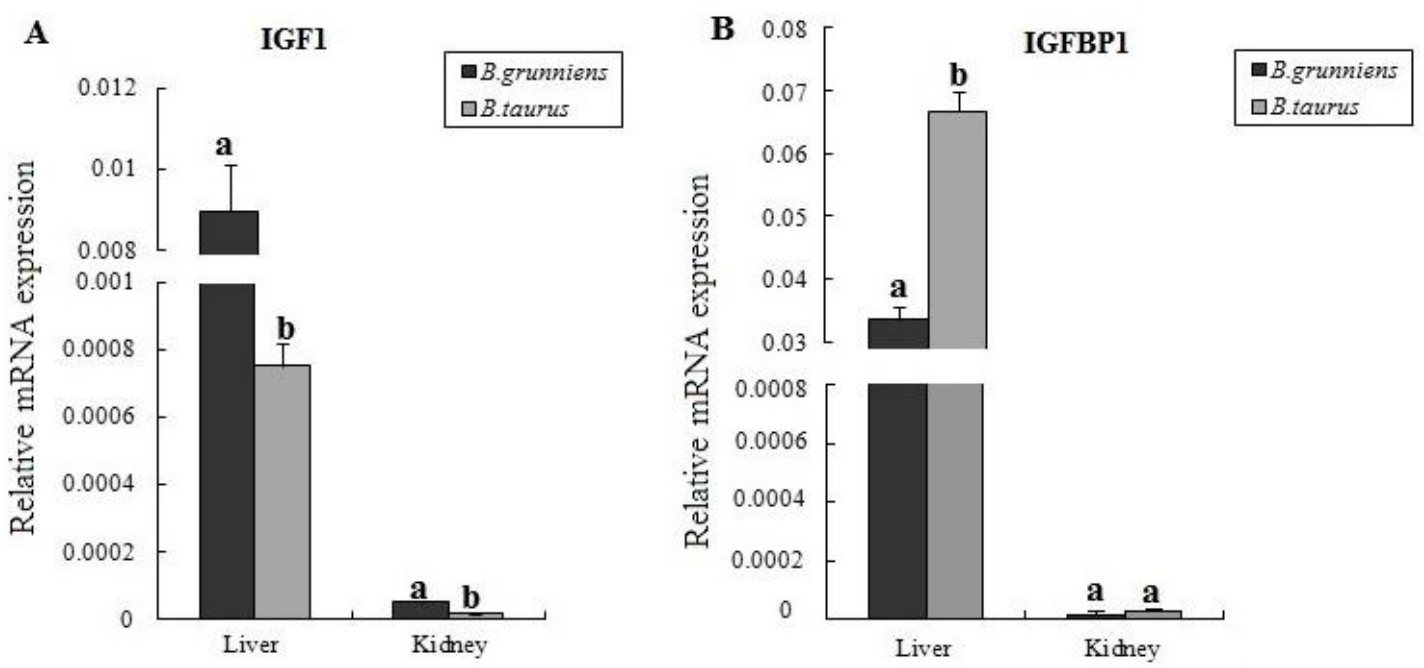

Figure 3. Expression levels of IGF-1 and IGFBP-1 in the livers and kidneys from B. grunniens $(\mathrm{n}=6)$ and $B$. taurus $(\mathrm{n}=6)$. Gene expression was normalized to the expression of $\beta$-actin. Different letters in the same tissue indicate difference between breeds $(\mathrm{p}<0.05$ ). IGF-1, insulin-like growth factor-1; IGFBP-1, insulin-like growth factor binding protein-1.

$\mathrm{Hu}, 2013)$. However, the availability of IGF-1 is regulated by IGFBPs, including IGFBP-1. Under the stimulus of low oxygen, IGFBP-1 expression can be upregulated; consequently, the availability of IGF-1 would be reduced, and IGFR pathway would be inhibited (Kajimura et al., 2005; Sun et al., 2011). However, little information is available on the variation in the expression patterns of IGF1 and IGFBP-1 between plateau and lowland animals that may be used to investigate the molecular mechanisms adapting to hypoxia for highland animals.

Yaks of the Qinghai-Tibet plateau live at high altitudes from 3,000 $\mathrm{m}$ to $5,000 \mathrm{~m}$ and have been exposed generation by generation to the hypoxic and cold environment. In this study, we compared the difference in the expression levels of IGF-1 and IGFBP-1 in liver and kidney tissues between yaks and lowland cattle. Results showed that both IGF-1 and IGFBP-1 were highly expressed in the liver than in the kidney in the same species (Figure 3), which is in agreement with the findings in previous reports (Ohlsson et al., 2009; Zhang et al., 2013). Comparison of the two breeds showed that the expression level of IGF-1 was higher $(p<0.05)$ in the tissues of yaks than that in cattle, indicating that IGF-1 may play an important role in the adaptation to the hypoxic environment. In the yak, the upregulated expression of IGF-1 can induce HIF1 expression, and hence, trigger various genes to express and regulate the cellular hypoxia response (Fukuda et al., 2002; Sutton et al., 2007). In addition, $I G F B P-1$ gene was expressed at a significantly higher level in the liver of cattle than that in yaks $(\mathrm{p}<0.05)$, but it had similar expression levels in the kidneys of both species. The IGFBP-1 is mainly synthesized in the liver, and lesser production of IGFBP-1 can ensure sufficient IGF-1 in circulation to bind to the IGFR to respond to hypoxic stress in yak. Moreover, the equal IGFBP-1 in yak indicates that IGFBP-1 might have other different physiological roles in yak.

In summary, we have cloned and characterized the cDNA sequences of IGF-1 and IGFBP-1 from yaks. The widespread distribution of IGF-1 and IGFBP-1 suggested that they play important roles in the growth and development in bovines. Moreover, the upregulated IGF-1 expression and downregulated IGFBP-1 expression are associated with hypoxia adaptive response. In our future studies we will investigate the molecular mechanism of $I G F-1$ and IGFBP-1 gene regulation associated with the adaptation to hypoxia to elucidate the differential expression.

\section{ACKNOWLEDGMENTS}

This work was supported by Graduate innovative projects of Southwest University for Nationalities (CX2014SZ75), and we thank all members of the Cell Engineering Lab.

\section{REFERENCES}

Annunziata, M., R. Granata, and E. Ghigo. 2011. The IGF system. Acta Diabetol. 48:1-9.

Cao, Y.-B., X.-Q. Chen, S. Wang, X.-C. Chen, Y.-X. Wang, J. Chang, and J.-Z. Du. 2009. Growth hormone and insulin-like growth factor of naked carp (Gymnocypris przewalskii) in lake Qinghai: Expression in different water environments. Gen. Comp. Endocrinol. 161:400-406.

Cao, Y.-B., X.-Q. Chen, S. Wang, Y.-X. Wang, and J.-Z. Du. 2008. Evolution and regulation of the downstream gene of hypoxiainducible factor-1 $\alpha$ in naked carp (Gymnocypris przewalskii) 
from lake Qinghai, China. J. Mol. Evol. 67:570-580.

Eliasz, S., S. Liang, Y. Chen, M. A. De Marco, O. Machek, S. Skucha, L. Miele, and M. Bocchetta. 2010. Notch-1 stimulates survival of lung adenocarcinoma cells during hypoxia by activating the IGF-1R pathway. Oncogene 29:2488-2498.

Fukuda, R., K. Hirota, F. Fan, Y. Do Jung, L. M. Ellis, and G. L. Semenza. 2002. Insulin-like growth factor 1 induces hypoxiainducible factor 1-mediated vascular endothelial growth factor expression, which is dependent on MAP kinase and phosphatidylinositol 3-kinase signaling in colon cancer cells. J. Biol. Chem. 277:38205-38211.

Hu, Q., T. Ma, K. Wang, T. Xu, J. Liu, and Q. Qiu. 2012. The Yak genome database: An integrative database for studying yak biology and high-altitude adaption. BMC Genomics 13:600.

Jones, J. I. and D. R. Clemmons. 1995. Insulin-like growth factors and their binding proteins: Biological actions. Endocr. Rev. 16:3-34.

Kajimura, S., K. Aida, and C. Duan. 2005. Insulin-like growth factor-binding protein-1 (IGFBP-1) mediates hypoxia-induced embryonic growth and developmental retardation. Proc. Natl. Acad. Sci. USA. 102:1240-1245.

Lee, P. D. K., L. C. Giudice, C. A. Conover, and D. R. Powell. 1997. Insulin-like growth factor binding protein-1: Recent findings and new directions. Exp. Biol. Med. 216:319-357.

Mehrhof, F. B., F.-U. Müller, M. W. Bergmann, P. Li, Y. Wang, W. Schmitz, R. Dietz, and R. von Harsdorf. 2001. In cardiomyocyte hypoxia, insulin-like growth factor-I-induced antiapoptotic signaling requires phosphatidylinositol-3-OHkinase-dependent and mitogen-activated protein kinasedependent activation of the transcription factor cAMP response element-binding protein. Circulation 104:2088-2094.

Murphy, C. T. and P. J. Hu. 2013. Insulin/insulin-like growth factor signaling in C. elegans. WormBook: The Online Review of C. elegans biology: 1.

Ohlsson, C., S. Mohan, K. Sjogren, A. S. Tivesten, J. Isgaard, O. Isaksson, J.-O. Jansson, and J. Svensson. 2009. The role of liver-derived insulin-like growth factor-I. Endocr. Rev. 30: 494-535.
Rajpathak, S. N., M. J. Gunter, J. Wylie-Rosett, G. Y. F. Ho, R. C. Kaplan, R. Muzumdar, T. E. Rohan, and H. D. Strickler. 2009. The role of insulin-like growth factor-I and its binding proteins in glucose homeostasis and type 2 diabetes. Diabetes/Metab. Res. Rev. 25:3-12.

Schmittgen, T. D. and K. J. Livak. 2008. Analyzing real-time PCR data by the comparative $\mathrm{C}_{\mathrm{T}}$ method. Nat. Protoc. 3:1101-1108.

Sugawara, J., S. I. Tazuke, L. F-Suen, D. R. Powell, F. Kaper, A. J. Giaccia, and L. C. Giudice. 2000. Regulation of insulin-like growth factor-binding protein 1 by hypoxia and $3^{\prime}, 5^{\prime}$-cyclic adenosine monophosphate is additive in HepG2 cells 1. J. Clin. Endocrinol. Metab. 85:3821-3827.

Sun, C.-F., Y. Tao, X.-Y. Jiang, andS.-M. Zou. 2011. IGF binding protein 1 is correlated with hypoxia-induced growth reduce and developmental defects in grass carp (Ctenopharyngodon idellus) embryos. Gen. Comp. Endocrinol. 172:409-415.

Sutton, K. M., S. Hayat, N.-M. Chau, S. Cook, J. Pouyssegur, A. Ahmed, N. Perusinghe, R. Le Floch, J. Yang, and M. Ashcroft. 2007. Selective inhibition of MEK1/2 reveals a differential requirement for ERK1/2 signalling in the regulation of HIF-1 in response to hypoxia and IGF-1. Oncogene 26:3920-3929.

Wei, D.-B., L. Wei, J.-M. Zhang, and H.-Y. Yu. 2006. Blood-gas properties of plateau zokor (Myospalax baileyi). Comp. Biochem. Physiol. Part A: Mol. Integr. Physiol. 145:372-375.

Weir, E., A. Tucker, J. Reeves, D. Will, and R. Grover. 1974. The genetic factor influencing pulmonary hypertension in cattle at high altitude1. Cardiovasc Res. 8:745-749.

Will, D. H., J. L. Hicks, C. S. Card, and A. F. Alexander. 1975. Inherited susceptibility of cattle to high-altitude pulmonary hypertension. J. Appl. Physiol. 38:491-494.

Yu, J., J. Li, S. Zhang, X. Xu, M. Zheng, G. Jiang, and F. Li. 2012. IGF-1 induces hypoxia-inducible factor $1 \alpha$-mediated GLUT3 expression through PI3K/Akt/mTOR dependent pathways in PC12 cells. Brain Res. 1430:18-24.

Zhang, S., Y. Zhao, X. Hu, Z. Liu, X. Chen, X. Chen, and J. Du. 2013. Distinct post-transcriptional regulation of $I g f b p l$ gene by hypoxia in lowland mouse and Qinghai-Tibet plateau root vole Microtus oeconomus. Mol. Cell. Endocrinol. 376:33-42. 